\title{
Prevalence of systemic inflammatory syndromes at a tertiary pediatric intensive care unit
}

\author{
Paulo R. A. Carvalho, ${ }^{1}$ Letícia Feldens, ${ }^{2}$ Elizabeth E. Seitz ${ }^{3}$ \\ Taís S. Rocha, ${ }^{4}$ Maria A. Soledade, ${ }^{5}$ Eliana A. Trotta ${ }^{6}$
}

\begin{abstract}
Objective: To assess the prevalence of systemic inflammatory syndromes on admission to a tertiary-care university pediatric intensive care unit (ICU), and relate this to length of hospital stay, risk of death and mortality rate.

Methods: Cross-sectional, prospective, observational study, including all patients admitted to the Hospital de Clínicas de Porto Alegre (HCPA) ICU between August $1^{\text {st }} 1999$ and July $31^{\text {st }} 2000$. Patient demographic variables were considered together with the risk of mortality on admission, co-morbidities, length of hospital stay and ICU outcome, in addition to variables that characterize the systemic inflammatory syndromes (systemic inflammatory response syndrome, sepsis, severe sepsis and septic shock).

Results: We studied 447 admissions of 388 patients; $54 \%$ were male, with a median age of 20 months. The prevalence of systemic inflammatory response syndrome (SIRS) was 68\%: 2/3 infectious (sepsis, severe sepsis or septic shock) and $1 / 3$ non-infectious. Risk of mortality scores for patients with infectious SIRS were higher than for those with non-infectious SIRS (6.75\% [P25=2.25 - P75=21.3] vs. 2.35\% [P25=1.1 - P75=6.7]; $\mathrm{p}=0)$ and increased according to SIRS severity $(2.9 ; 10.85,43.9 \% ; \mathrm{p}<0.05)$. The observed mortality was $12 \%$ for patients with SIRS and $5.8 \%$ for those without SIRS $(p=0.057)$; the observed mortality for infectious SIRS was $14.9 \%$ and for non-infectious $6.3 \%(p=0.041)$. The period spent in ICU for infectious SIRS was longer than for non-infectious cases: 3 days ( $P 25=2-P 75=7)$ vs. 2 days $(P 25=1.5-P 75=4) ; p=0.006$.

Conclusions: The prevalence rate of patients with systemic inflammatory response syndrome upon admission to HCPA pediatric intensive care unit was elevated, with a predominance of infectious syndromes, responsible for longer stays, increased risk of mortality and increased mortality of patients during the period evaluated.
\end{abstract}

J Pediatr (Rio J). 2005;81(2):143-8: Systemic inflammatory response syndrome, sepsis, severe sepsis, septic shock, prevalence, mortality, intensive care, children.

\section{Introduction}

The clinical criteria for and concepts of sepsis introduced in the last decade allowed for a more appropriate classification of inflammatory events in intensive care unit (ICU) patients.

1. PhD. Adjunct professor, Universidade Federal do Rio Grande do Sul (UFRGS), Porto Alegre, RS. Assistant intensive care physician PICU, Hospital de Clínicas de Porto Alegre (HCPA), Porto Alegre, RS, Brazil. 2. Resident physician, HCPA, Porto Alegre, RS, Brazil.

3. MSc., Pontifícia Universidade Católica do Rio Grande do Sul (PUCRS), Porto Alegre, RS, Brazil.

4. MSc., UFRGS, Porto Alegre, RS, Brazil.

5. Intensive care physician, PICU, HCPA, Porto Alegre, RS, Brazil.

6. Chief of the PICU, HCPA, Porto Alegre, RS, Brazil. Adjunct professor, UFRGS, Porto Alegre, RS, Brazil.

Manuscript received Sep 02 2004, accepted for publication Dec 222004

Suggested citation: Carvalho PR, Feldens L, Seitz EE, Rocha TS, Soledade MA, Trotta EA. Prevalence of systemic inflammatory syndromes at a tertiary pediatric intensive care unit. J Pediatr (Rio J). 2005;81:143-8.
Unarguably, these criteria and concepts brought benefits to the understanding and management of these clinical pictures, and also to the comparison of results involving new therapies for inflammatory syndromes. ${ }^{1}$

The diagnostic criteria for systemic inflammatory response syndrome (SIRS) published in 1991 are still questioned and discussed as to their sensitivity and specificity, mainly with regard to pediatric patients. In this age group, the concept of SIRS has not been properly validated, especially regarding the pattern of physiological variables in different pediatric segments (heart rate and respiratory frequency, arterial blood pressure and urinary output). Some publications show SIRS criteria adapted for use in children ${ }^{2-4}$ and employed in the scarce pediatric case reports, which acknowledgedly lack specificity. 2,3

Systemic inflammatory syndromes of infectious etiology or septic syndromes (sepsis, severe sepsis and 
septic shock) are quite common in ICUs, being the cause of death in up to $50 \%$ of the cases. ${ }^{5-8}$ Both the morbidity and mortality of these syndromes have risen in the last few decades because critically ill patients and those at quite an advanced stage of the disease have been increasingly treated. ${ }^{2}$

The aim of this study was to assess the prevalence rates of systemic inflammatory syndromes on the admission of patients to a pediatric teaching ICU, in addition to evaluating the length of stay in the ICU and the mortality rate of these patients within a one-year period.

\section{Patients and methods}

Between August 1st, 1999 and July 31st, 2000, we carried out a cross-sectional, prospective and observational study including patients admitted to the pediatric intensive care unit (PICU) of Hospital de Clínicas de Porto Alegre.

The Hospital de Clínicas de Porto Alegre is a general teaching hospital affiliated with Universidade Federal do Rio Grande do Sul, with 110 pediatric beds, and whose PICU has 13 beds for tertiary and multidisciplinary care of clinical and surgical patients, except for those with trauma, heart and brain surgeries, and neonatal diseases.

In this study, the following variables were considered: sex, age, reason for admission to the ICU, place of origin, risk of death based on the PRISM (Pediatric Risk of Mortality) score obtained in the first 24 hours after ICU admission, ${ }^{9}$ comorbidities, results of the blood test and/or cerebrospinal fluid test performed on ICU admission, length of stay in the ICU and outcome, in addition to clinical variables that characterize the inflammatory syndromes analyzed: $1-3,8,10,11$ (a) Systemic inflammatory response syndrome (SIRS) - characterized by two or more of the following conditions: (a) (axillary) temperature $>37.5^{\circ} \mathrm{C}$ or $<36^{\circ} \mathrm{C}$; (b) heart rate $>160 \mathrm{bpm}$ in infants and $>150 \mathrm{bpm}$ in children, or $>2$ SD above the normal values for age; (c) respiratory frequency $>60 \mathrm{mpm}$ in infant and $>50 \mathrm{mpm}$ in children, or $>2 \mathrm{SD}$ above the normal values for age; (d) total leukocyte count $>12,000$ cells $/ \mathrm{mm}^{3},<4,000$ cells $/ \mathrm{mm}^{3}$, or $>10 \%$ band forms. Sepsis - characterized by confirmed (positive culture) or highly suspected infection (evidence of infectious focus) combined with two or more of the conditions considered for SIRS. Severe sepsis characterized as sepsis associated with organ dysfunction, hypoperfusion or hypotension (systolic blood pressure $<10$ th percentile for age). Septic shock - characterized as sepsis associated with hypotension (systolic blood pressure < 10th percentile for age) even after appropriate volume resuscitation, plus the presence of systemic perfusion disorders.

The descriptive statistics is expressed as median for the measures of central tendency and as interquartile range for the measures of dispersion (P25 and P75). Comparative analyses were made using the chi-square test for categorical variables. The Mann-Whitney and Kruskall-Wallis tests were used for continuous variables without normal distribution, and Dunn's test was used for multiple comparisons between the means. A $p$ value $<0.05$ was regarded as statistically significant. The SPSS 10.0 program was used for the statistical analysis.

The study protocol was approved by the Research and Ethics Committee of Hospital de Clínicas de Porto Alegre. No written consent form had to be signed because it was an epidemiological and observational study that included all PICU patients admitted between August 1st, 1999 and July 31 st, 2000.

\section{Results}

A total of 447 ICU admissions of 388 patients were assessed. Fifty-four percent of the patients were male, with ages between one month and 17 years (median of 20 months). The risk of death calculated by the PRISM score at the time of admission showed a median of $3.5 \%$ (P25 = 1.7 and P75 = 12.9). The origin of patients was distributed among clinical units (31\%) and surgical units $(11 \%)$ of the hospital, emergency room (30\%) and transfers from other hospitals $(28 \%)$.

The main reasons for ICU admission were respiratory failure (39\%), postoperative care (18\%) and hemodynamic instability ( $12 \%)$. Of admitted patients, $36 \%$ did not have any comorbidities; among 278 patients with previous history of disease, $15.5 \%$ had lung diseases, $13 \%$ liver diseases, $12 \%$ cancer and $11.5 \%$ genetic diseases.

The prevalence rate of SIRS on admission was $68 \%$ $(n=304)$ (Figure 1$)$. SIRS apparently unrelated to infection occurred in $36 \%$ of patients $(n=110)$ diagnosed with SIRS, whereas infectious or septic syndromes (sepsis, severe sepsis and septic shock) were observed in the remaining $64 \%(n=194)$ (Figure 2$)$.

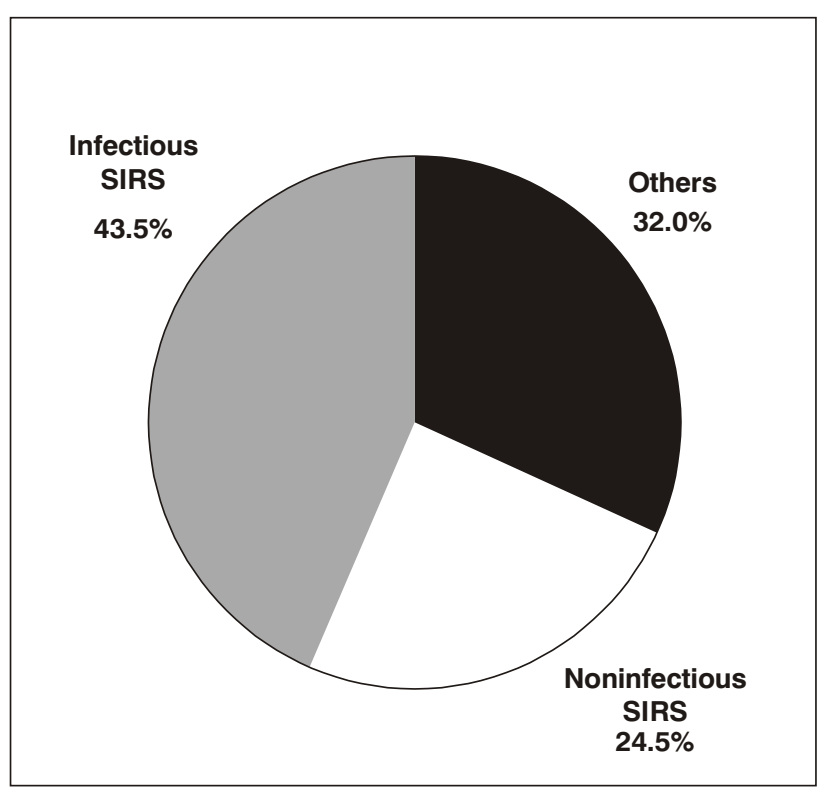

SIRS = systemic inflammatory response syndrome.

Figure 1 - Distribution of admission diagnosis $(n=447)$ 


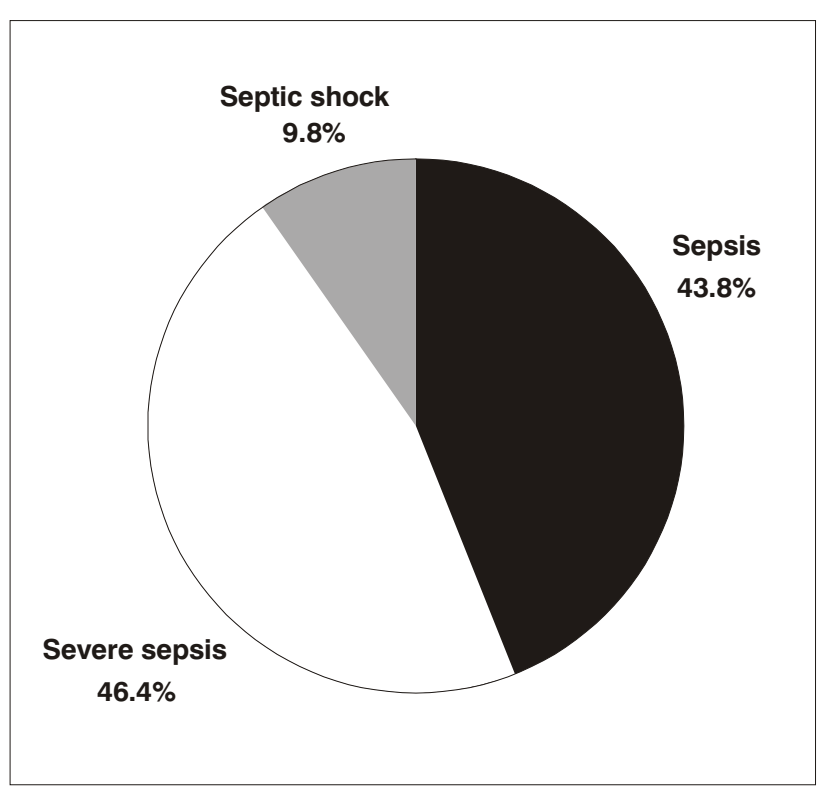

Figure 2 - Distribution of septic syndromes on admission ( $\mathrm{n}=$ 194)

The diagnosis of the syndrome was predominantly based on the presence of two $(n=138)$ or three $(n=110)$ of the four clinical criteria recommended. The four criteria were found concomitantly in $15 \%$ of SIRS patients $(n=46)$. "Increased heart rate" occurred in $85.5 \%$ of SIRS patients, "abnormal leukocyte count" in $77 \%$, "abnormal temperature" in $62 \%$ and "increased respiratory frequency" in $47.5 \%$ (Figure 3 ).

The identification of the microbial agent in blood or CSF samples obtained on admission occurred in 63 patients ( $14 \%$ of admissions) $-84 \%$ in patients with infectious SIRS and $16 \%$ in patients without SIRS. Among the patients with infectious SIRS, the identification of the

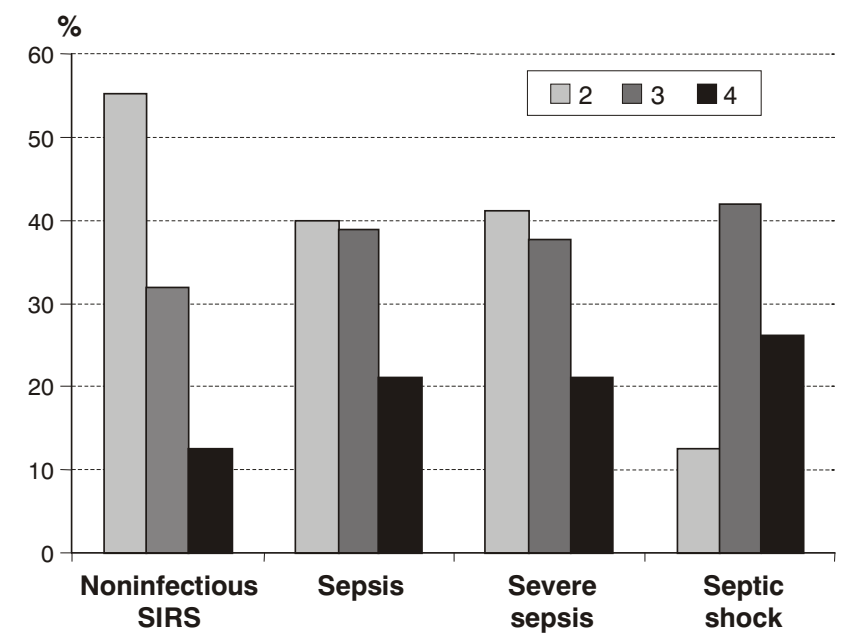

Figure 3 - Distribution of the number of criteria according to each diagnosis on admission $(n=447)$ microbial agent occurred in only $26 \%$. Of the 10 patients who did not meet the criteria for SIRS, but who showed signs of the microbial agent, only three did not have any clinical evidence of infection.

The risk of death calculated by the PRISM score on admission was statistically higher in SIRS patients ( median $=4.15 \%[\mathrm{P} 25=1.85$ and $\mathrm{P} 75=15.5]$ ) compared to those without SIRS (median $=2.65 \%[$ P25 $=1.1$ and $\mathrm{P} 75=8.3] ; \mathrm{p}=0$ ); likewise, it was statistically higher in patients with infectious SIRS (median $=6.75 \%[$ P25 $=2.25$ and $\mathrm{P} 75=21.3]$ ) compared to noninfectious SIRS ( median $=2.35 \%[$ P25 $=1.1$ and P75 $=6.7] ; \mathrm{p}=0)$, $($ Table $1)$. In infectious inflammatory syndromes, the risk of death increased with the severity of the disease (Table 2).

Among the sampled patients, 44 died during the study period. Similarly to what occurred with the predicted mortality rate, observed mortality varied according to the diagnosis of the syndrome on admission: $12 \%(n=36)$ in SIRS patients and $5.8 \%(n=8)$ in those without SIRS $(p=0.057)$. In infectious SIRS, mortality amounted to $14.9 \%(n=29)$, whereas in noninfectious SIRS, it corresponded to $6.3 \%$ $(n=7)(p=0.041)$.

The median age in the group of SIRS patients was 24 months $(P 25=5.25$ and P75 $=72.5)$, greater than that of the group without SIRS (median $=12$ months $[$ P25 $=4$ and $\mathrm{P} 75=60] ; \mathrm{p}=0.012$ ). Among SIRS patients, the median age of the group with noninfectious SIRS was 39 months $(P 25=16.5$ and P75 = 89.5), way above that of the group with infectious SIRS (median $=16$ months $[$ P25 $=4$ and P75 $=61] ; \mathrm{p}=0$ ).

The length of stay in the ICU ranged from $<1$ to 72 days ( median $=3$ days $[$ P25 $=2$ and P75 = 6]). The same median ( 3 days) was observed in patients with SIRS (P25 = 2 and P75 = 6) and without SIRS (P25 = 2 and P75 = 6) $(p=0.184)$. However, when the groups were compared according to the etiology of SIRS, the patients with infectious SIRS had a median length of stay in the ICU of 3 days $(P 25=2$ and P75 = 7), statistically higher than that of patients with noninfectious SIRS ( median $=2$ days $[$ P25 $=1.5$ and $\mathrm{P} 75=4] ; \mathrm{P}=0.006)$. With regard to patient outcome, those patients who failed to survive in the ICU $(n=44)$ had a median length of stay of 5.5 days ( $P 25=1$ and P75 = 13.75), significantly higher than those who survived, who had a median stay of 3 days $(P 25=2$ and P75 $=5)(p=0.031)$.

\section{Discussion}

The assessment of prevalence on admission partially shows the occurrence of systemic inflammatory syndromes in the ICU, as the cumulative incidence rate of septic syndromes, for instance, is higher after admission, due to the infections acquired in the ICU, which result in greater morbidity and mortality and higher costs for hospitals. ${ }^{12-15}$ On the other hand, the type of ICU interferes with the rate of SIRS on admission - in those predominantly surgical or trauma units, the prevalence of noninfectious SIRS is higher on admission, unlike clinical ICUs, where infectious SIRS predominates. ${ }^{11,16-18}$ 
Table 1 - Pediatric Risk of Mortality (PRISM) on admission according to the diagnosis of the syndrome

\begin{tabular}{lccc}
\hline & & \multicolumn{2}{c}{ PRISM on admission } \\
\cline { 3 - 4 } Diagnosis of the syndrome & $\mathbf{n}$ & Median (\%) & Interquartile range \\
\hline Without SIRS & 143 & 2.65 & $\mathrm{P} 25=1.10$ and P75 $=8.30$ \\
With SIRS & 304 & 4.15 & $\mathrm{P} 25=1.85$ and P75 $=15.50 *$ \\
$\quad$ Noninfectious SIRS & 110 & 2.35 & $\mathrm{P} 25=1.10$ and P75 $=6.70$ \\
$\quad$ Infectious SIRS & 194 & 6.75 & $\mathrm{P} 25=2.25$ and P75 $=21.30^{\dagger}$ \\
\hline
\end{tabular}

Mann-Whitney Test:

* statistics $p=0$.

$\dagger$ statistics $p=0$.

SIRS = systemic inflammatory response syndrome.

Table 2 - Pediatric Risk of Mortality (PRISM) on admission according to diagnosis of septic syndrome

\begin{tabular}{lccc}
\hline & & \multicolumn{2}{c}{ PRISM on admission } \\
\cline { 3 - 4 } Diagnosis of the syndrome & $\mathbf{n}$ & Median (\%) & Interquartile range \\
\hline Sepsis & 85 & 2.95 & $\mathrm{P} 25=1.75$ and $\mathrm{P} 75=7.25$ \\
Severe sepsis & 90 & 10.85 & $\mathrm{P} 25=5.10$ and $\mathrm{P} 75=30.15$ \\
Septic shock & 19 & 43.90 & $\mathrm{P} 25=8.70$ and $\mathrm{P} 75=86.90 *$ \\
\hline
\end{tabular}

* Satistically significant difference (Kruskall-Wallis Test and Dunn; $p<0.05$ ).

In the present cross-sectional study, in which the sample is characterized by low admission of patients from the emergency room $(30 \%)$, the predominance of infectious syndromes on admission over noninfectious SIRS may be ascribed to the predominantly clinical characteristic of the unit and to the fact that it is a referral center for several chronic diseases, where the conditions for the acquisition of infection are less related to community-acquired diseases. ${ }^{19}$

There is a paucity of studies about the incidence or prevalence of SIRS in pediatric patients; therefore, it is not possible to compare the results obtained from the analyzed patient population. One of the most widely known epidemiological studies, the one conducted by Proulx et al. ${ }^{10}$ in a Canadian PICU, revealed a cumulative SIRS incidence of $82 \%$, with a median length of stay in the ICU of 2 days. The study was carried out in a clinical-surgical ICU, where bone marrow transplant recipients were not allowed and where there were few burn patients. In the present study, a $68 \%$ SIRS prevalence on admission, whose comparison with that study is also limited due to the study design used, portrays a tertiary teaching PICU, to which patients with trauma, heart and brain surgeries and neonatal diseases are not referred, but where two thirds of the patients have comorbidities. Thus, the type of ICU explains the higher prevalence of infectious SIRS -43.5 vs. $24.5 \%$ (Figure 1), since a lot of patients with chronic and debilitating diseases are admitted. If we consider the cumulative incidence of infectious SIRS, the infectious type often prevails over the noninfectious one, since septic syndromes have a predominantly nosocomial etiology. ${ }^{11,16-18}$

In the Canadian study, infectious SIRS corresponded to less than $30 \%$. As to the distribution of infectious inflammatory syndromes, that study showed an incidence of $79.3 \%$ for sepsis, $13.8 \%$ for severe sepsis and $6.9 \%$ for septic shock, for the total length of ICU stay. A retrospective study carried out by Sáez-Llorens et al. ${ }^{11}$ detected $18 \%$ of infectious SIRS in a population of 4,529 patients admitted to a Panamanian pediatric ICU, whose incidence rates were the following: $21 \%$ for sepsis, $61 \%$ for severe sepsis and $18 \%$ for septic shock. In the present study, whose prevalence rate for infectious SIRS on admission was 43.5, the distribution of septic syndromes was $43.8,46.4$ and $9.8 \%$ for sepsis, severe sepsis and septic shock, respectively. The different distributions of septic syndromes in the analyzed studies may suggest difficult access to the ICU, difficulty in 
establishing a diagnosis or early intervention, or may be related to the nature of the disease.

The diagnosis of systemic inflammatory syndromes, although in compliance with the criteria recommended by the Consensus Conference on Sepsis ${ }^{1}$ in 1991, adapted to the pediatric population, ${ }^{2,3}$ is still arguable due to its low specificity. In the present study, the presence of two, three or four criteria for definition of SIRS did not prove useful in improving the specificity of clinical diagnoses of inflammatory syndromes. In the case of septic syndromes, the mere identification of the etiologic agent may lead to a definitive diagnosis even if in over $30 \%$ of the situations the cultures yield negative results. ${ }^{13,14}$ Due to these diagnostic limitations, the recent 2001 International Conference on Sepsis included a list of clinical and laboratory diagnostic criteria that may help to consider infected patients as "septic-looking."20 In the study conducted by Proulx et al. ${ }^{10}$ only $5 \%$ of the patients had at least one positive blood culture during their stay in the ICU. Considering that less than $30 \%$ of analyzed patients had septic syndrome, the etiologic agent was identified only in $15 \%$ of the cases. In the study undertaken by Sáez-Llorens et al., ${ }^{11}$ which added the erythrocyte sedimentation rate and serum C-reactive protein concentration to the recommended criteria, infectious syndromes were bacteriologically confirmed in $26 \%$ of the cases. In the present study, only $15 \%$ of the patients met four classic criteria for SIRS and, of the whole sample, only $14 \%$ had the microbial agent identified, mostly patients with septic syndromes (only $26 \%$ of positivity in the infectious SIRS group). Surprisingly, the microbial agent was identified in 10 patients who did not meet the diagnostic criteria for SIRS, reinforcing the assumption of low sensitivity and specificity of the initially recommended criteria.

In the present study, the risk calculated as observed risk of death in patients without SIRS was quite similar to that of patients with noninfectious SIRS. We also observed a higher prevalence of infectious SIRS in young patients, who are certainly more susceptible to infectious agents. Therefore, the infectious etiology seems to be a determining factor in the increase of prevalence and mortality from SIRS in PICUs, which has been confirmed by other studies that associate systemic infections with higher mortality in ICU.10,17

The present study revealed a $15 \%$ mortality in patients with septic syndrome, which was twice as high as that of patients with noninfectious SIRS (6.3\%), and way below that which was observed in the study conducted by SáezLlorens et al., (39\%). ${ }^{11}$ In the study carried out by Proulx et al., which related the mortality of inflammatory syndromes with the presence of multiple organ dysfunction syndrome, the mortality rate for septic syndromes was $32 \% .10$

The study design did not allow establishing a causeeffect relationship between the diagnosis of the syndrome on admission and the length of stay in the ICU, since the development of the initial situation and the clinical intercurrent events that might arise from it during the stay in the ICU would be highly diverse and have an unpredictable outcome. We may infer that infectious events demand a long-term specific treatment and include occasional complications, as actually occurred in the analyzed patient population, where the length of stay in the ICU of patients with infectious syndromes was almost twice that of patients with noninfectious SIRS. Likewise, patients who died had spent a longer time in the ICU than those who survived.

Our conclusion is that the prevalence of patients with systemic inflammatory syndromes on ICU admission was high in the analyzed cases, with predominance of infectious syndromes (sepsis, severe sepsis and septic shock) over other types. These syndromes are responsible for a longer stay in the ICU, for a higher risk of death and for a higher mortality rate among ICU patients.

\section{References}

1. American College of Chest Physicians / Society of Critical Care Medicine Consensus Conference: Definitions for sepsis and organ failure and guidelines for the use of innovative therapies in sepsis. Crit Care Med. 1992;20:864-74.

2. Leclerc F, Martinot A, Fourier C. Definitions, risk factors, and outcome of sepsis in children. In: Tibboel D, van der Voort E, eds. Update in intensive care and emergency medicine 25. Intensive care in childhood. A challenge to the future. SpringerVerlag: Berlin: 1996. Pp. 227-38.

3. Hayden WR. Sepsis terminology in Pediatrics. [Letter]. J Pediatr. $1994 ; 124: 657-8$.

4. Jafari HS, McCracken GH. Sepsis and septic shock: a review for clinicians. Pediatr Infect Dis J. 1992;11:739-48.

5. Jacobs RF, Sowell MK, Moss M, Fiser DH. Septic shock in children: bacterial etiologies and temporal relationships. Pediatr Infect Dis J. 1990;9:196-200.

6. Carcillo JA, Davis AL, Zaritsky A. Role of early fluid resuscitation in pediatric septic shock. JAMA. 1991; 266:1242-5.

7. Bone RC. The pathogenesis of sepsis. Ann Intern Med. 1991;115: 457-69.

8. Tran DD, Groeneveld AB, van der Meulen J, Nauta JJ, Strack van Schijndel RJ, Thijs LG. Age, chronic disease, sepsis, organ system failure, and mortality in a medical intensive care unit. Crit Care Med. 1990;18:474-9.

9. Pollack MM, Ruttimann UE, Getson PR. Pediatric Risk of Mortality (PRISM) score. Crit Care Med. 1988;16:1110-6.

10. Proulx F, Fayon M, Farrell CA, Lacroix J, Gauthier M. Epidemiology of sepsis and multiple organ dysfunction syndrome in children. Chest. 1996;109:1033-7.

11. Sáez-Llorens X, Vargas S, Guerra F, Coronado L. Application of new sepsis definitions to evaluate outcome of pediatric patients with severe systemic infections. Pediatr Infect Dis J. 1995; 14:557-61.

12. Brun-Buisson C, Roudot-Thoraval F, Girou E, Grenier-Sennelier $C$, Durand-Zaleski I. The costs of septic syndromes in the intensive care unit and influence of hospital-acquired sepsis. Intensive Care Med. 2003;29:1464-71.

13. Alberti C, Brun-Brisson C, Burchardi H, Martin C, Goodman S, Artigas $A$ et al. Epidemiology of sepsis and infection in ICU patients from an international multicentre cohort study. Intensive Care Med. 2002;28:108-21.

14. Padkin A, Goldfrad C, Brady AR, Young D, Black N, Rowan K. Epidemiology of severe sepsis occurring in the first $24 \mathrm{hrs}$ in intensive care units in England, Wales, and Northern Ireland. Crit Care Med. 2003;31:2332-8.

15. Angus DC, Linde-Zwirble WT, Lidicker J, Clermont G, Carcillo J, Pinsky MR. Epidemiology of severe sepsis in the United States: analysis of incidence, outcome, and associated costs of care. Crit Care Med. 2001;29:1303-10. 
16. Brun-Bruisson C, Doyon F, Carlet J, Dellamonica P, Gouin F, Lepoutre $A$. et al. Incidence, risk factors, and outcome of severe sepsis and septic shock in adults. JAMA. 1995;274:968-74.

17. Bone RC. Toward an epidemiology and natural history of SIRS (systemic inflammatory response syndrome). JAMA. 1992; 268:3452-5.

18. Rangel-Fausto MS, Pittet D, Costigan M, Hwang T, Davis CS, Wenzel RP. The natural history of the systemic inflammatory response syndrome (SIRS). JAMA. 1995;273:117-23.

19. Siegman-Igra $Y$, Fourer, Orni-Wasserlauf R, Golan $Y$, Noy $A$, Schwartz D et al. Reappraisal of community-acquired bacteremia: a proposal of a new classification for the spectrum of acquisition of bacteremia. Clin Infect Dis. 2002;34:1431-9.
20. Levy MM, Fink MP, Marshall JC, Abraham E, Angus D, Cook D. et al. 2001 SCCM/ESICM/ACCP/ATS/SIS International Sepsis Definitions Conference. Crit Care Med. 2003;31:1250-6.

\section{Correspondence:}

Paulo Roberto Antonacci Carvalho

Avenida Encantado, 249

CEP 90470-420 - Porto Alegre, RS

Brazil

Fax: +55 (51) 3330.6334

E-mail: carvalho.conex@uol.com.br 